\title{
Further Results on Exponentially Robust Stability of Uncertain Connection Weights of Neutral-Type Recurrent Neural Networks
}

\author{
Wenxiao Si $\mathbb{D}$, Tao Xie $\mathbb{D}$, and Biwen Li $\mathbb{D}$ \\ Hubei Normal University, Huangshi 435002, Hubei, China \\ Correspondence should be addressed to Tao Xie; 1044806961@qq.com
}

Received 25 June 2021; Revised 11 August 2021; Accepted 30 August 2021; Published 23 September 2021

Academic Editor: Yue Song

Copyright (C) 2021 Wenxiao Si et al. This is an open access article distributed under the Creative Commons Attribution License, which permits unrestricted use, distribution, and reproduction in any medium, provided the original work is properly cited.

\begin{abstract}
Further results on the robustness of the global exponential stability of recurrent neural network with piecewise constant arguments and neutral terms (NPRNN) subject to uncertain connection weights are presented in this paper. Estimating the upper bounds of the two categories of interference factors and establishing a measuring mechanism for uncertain dual connection weights are the core tasks and challenges. Hence, on the one hand, the new sufficient criteria for the upper bounds of neutral terms and piecewise arguments to guarantee the global exponential stability of NPRNN are provided. On the other hand, the allowed enclosed region of dual connection weights is characterized by a four-variable transcendental equation based on the preceding stable NPRNN. In this way, two interference factors and dual uncertain connection weights are mutually restricted in the model of parameter-uncertainty NPRNN, which leads to a dynamic evolution relationship. Finally, the numerical simulation comparisons with stable and unstable cases are provided to verify the effectiveness of the deduced results.
\end{abstract}

\section{Introduction}

Since recurrent neural networks (RNNs) have the ability of parallel processing, distributed information storage, and associative deep learning, a series of neural networks such as Hopfield neural network, Cohen-Grossberg neural network, cellular neural network, BAM neural network, high-order cellular neural network, and shunt inhibition neural network, which are the typical representatives of RNNs, have attracted extensive attention over the years. Accordingly, with the in-depth study of RNNs, it can be seen that the stability is a forerunner condition for the multifarious practical applications. Hence, the research on the stability of the system is becoming more and more abundant [1-14], such as asymptotic stability [1], exponential stability [2-4], multistability [5], synchronization [6], dissipativity $[3,7,8]$, region stability [9], memristorbased dynamic behavior stability [10], and exponential Lagrange stability $[11,12]$. Additionally, in terms of the widespread application fields such as the visual optimization, image processing, language recognition, associative memory, and other fields, the stability of RNNs has become an indispensable dynamical behavior characteristic which must be further considered.

Exponential stability and robustness, as for the classical dynamic behaviors of neural networks, have been studied extensively in the past few years $[2,3,13-22]$. On the one hand, global exponential stability reveals the superiority that it guarantees the system can be fleetly stable at the equilibrium point with an exponential decay rate, which deservedly leads to a rapid stabilization and dramatically saves the response time. Furthermore, the decay rate value can be intuitively captured [14]. On the other hand, due to the tunable flexibility and broad applicability, the robustness is always endowed with different meanings in disparate practical scenarios. Taking [13] as an example, a natural question is raised and deeply explored: how much the interference intensity can a disturbed system bear to realize the stability again on the basis of its original stability, which implies the exact connotation of the robustness studied in this paper. In addition, the emerging literatures [1-23] have also indirectly confirmed that various generalized stability behaviors of the neural networks are inevitably and immensely subject to the category and quantity of disturbances, 
for instance, time delays [1-8], stochastic disturbances $[8,13,15]$, parameter disturbances [9], piecewise constant arguments [14], neutral terms [22], and Markov switching $[12,23]$. Hence, the subsequent perturbations will be attached to RNNs to further examine and guarantee the robustness of global exponential stability (RoGES) of RNNs.

Neutral-type RNNs refer to RNNs with the neutral terms appeared in the derivative part, which makes the nonlinear perturbations affect not only the current states but also the states of derivative part. At present, neural networks with neutral terms have been applied in practice, for instance, the electrical interconnect and the electromagnetic interference design in digital computers are used as the specific physical application background for delayed neutral-type differential equations [24]. In combination with the statements mentioned above and some existing literatures, two species of exogenous interferences that diffusely appear in the modern engineering should be taken into consideration for neutraltype RNNs. On the one hand, due to the pervasive restrictions of the switching speed of each node of the neural networks, time delay is such a kind of inevitable interference element that it has been widely applied to various neural networks. And, piecewise constant argument studied here is another form of time delay, which unifies the hysteresis and advance. The investigation of RNNs subject to piecewise arguments is a breakthrough in literature [14], and the sufficient conditions for the exponential stability of a class of RNNs with piecewise arguments are given by constructing Lyapunov functions. At present, there are also some literatures further exploring the properties of the systems equipped with piecewise arguments $[15,25,26]$. On the other hand, it can be found that the parameter-intensity of the connection weight matrix is a crucial index affecting the stability of the systems [16-21, 27, 28]. Firstly, since parametric uncertainty was introduced in $[16,17,27,28]$, diverse forms of uncertain connection weights were applied to neural networks. Later, Zhu and Shen [18] visually depicted the boundary of the uncertain connection weights by the enclosed curve graph method and provided the sufficient conditions for RNNs disturbed by uncertain parameters to achieve the global exponential stability. Furthermore, time delays and random perturbations are additionally attached on RNNs in [19-21]. At present, uncertain connection weights have been applied to various fields, such as medicine, information transmission, and operations' research and planning [29-32]. It follows that the intensity of the connection weights is a highly mutable but immensely critical indicator.

By virtue of the existing literatures in the past decades, the stability of neutral-type RNNs with piecewise arguments or other time delays is explored by [22, 33-37] and some relatively mature methods have been widely used, such as the Euler-Maclaurin method, one-leg method, block boundary value method, and multidomain Legendre spectral collocation method. In addition, the investigations of RNNs instead of neutral-type RNNs with uncertain connection weights also have been carried out in $[16-21,27,28]$. However, there is hardly any studies aiming for the RoGES of the recurrent neural network with neutral terms and piecewise constant arguments (NPRNN) with uncertain connection weights. Hence, it is a notable problem that how much the parameter intensity of dual connection weights can a disturbed NPRNN endure to remain stable again on the basis of the original stable NPRNN.

Motivated by the above statements, here we investigate the RoGES of the NPRNN with uncertain connection weights. Naturally, the ultimate goal herein is to quantify the boundary values of the connection weight matrices on the basis of stable NPRNN. Hence, the main contributions are as follows. (1) The upper bounds of piecewise arguments and neutral terms that NPRNN can maintain stability are established. We solve the tricky neutral terms newly appeared in the derivative part of RNN by constructing a Lipschitz condition, and then, we obtain the upper bound of the neutral terms by solving a univariate transcendental equation. Afterwards, by fixing an appropriate value of neutral terms, the upper bound of piecewise arguments is settled by solving several different binary transcendental equations. (2) An enclosed curve about dual connection weights $(\sigma$ and $\lambda$ ) is acquired by solving the newly established four-element (two disturbances and two uncertain connection weights) transcendental equation on the basis of the preceding stable NPRNN, which is the core significant results of this paper (more details can be seen in Remarks 2 and 3). (3) By virtue of the above two upper bounds of the interference factors and the characterized enclosed curve about the dual-parameter intensity, the simulation results indicate that, as long as one of the interference values exceeds the derived bounds, the preceding stable NPRNN will be unstable, which also intuitively confirmed the validity of deduced results. Based on what has been established above, these four interference elements are mutually restricted and the relationship among these factors established by a four-element transcendental equation is dynamic.

The rest of the paper is as follows. The preliminaries and the model descriptions are included in Section 2. Then, the feasible threshold values of the piecewise arguments, the neutral terms, and the uncertain connection weights to achieve the RoGES of the parameter-uncertainty NPRNN are discussed in Section 3. Accordingly, the simulation comparisons to verify the validity of the deduced results are shown in Section 4. Finally, a brief conclusion and some feasible prospects for future work are given in Section 5.

\section{Problem Formulation}

Based on this paper, denote $\mathbf{N}$ as the natural number set and $\mathbf{R}$ as the real number set. And, for any constant $n$, denote $\underline{n}=\{1,2, \ldots, n\}$. $\mathbf{Z}^{+}$is the positive integer set. $\mathbb{R}^{n}$, $\mathbb{R}^{n \times m}$, and $\mathbb{R}^{+}$stand for $n$-dimensional Euclidean space, $n \times m$ real matrix space, and positive real space, respectively. Let $\|\cdot\|$ be the Euclidean norm, and the operator norm of matrix $A$ is defined as $\|A\|=\sup \{\|A x\|:\|x\|=1\}$. Denote two piecewise constant argument real-value sequences $\left\{\theta_{i}\right\}$ and $\left\{\eta_{i}\right\}$, such that $\theta_{i}<\eta_{i}<\theta_{i+1}, \theta_{i} \longrightarrow \infty$ when $i \longrightarrow \infty$ and $i \in \mathbb{N}$. 
Consider a NPRNN as

$$
\left\{\begin{array}{l}
d[v(t)-G(v(t))]=[-A v(t)+B f(v(t))+C g(v(\gamma(t)))] \mathrm{d} t, \\
v\left(t_{0}\right)=v_{0} \in \mathbb{R}^{n},
\end{array}\right.
$$

where $v(t)=\left(v_{1}(t), \ldots, v_{n}(t)\right) \in \mathbb{R}^{n}$ represents the state vector of the neurons, $G(v(t)): \mathbb{R}^{n} \longrightarrow \mathbb{R}^{n}$ is the neutraltype function of $v(t) \in \mathbb{R}^{n}, A=\operatorname{diag}\left\{a_{1}, \ldots, a_{n}\right\}$ is a selffeedback connection weight matrix, $B=\left(b_{i j}\right)_{n \times n}$ and $C=$ $\left(c_{i j}\right)_{n \times n}$ are the connection weight matrices of $f(v(t))$ and $g(v(\gamma(t)))$, respectively, where $A, B, C \in \mathbb{R}^{n \times n}, f(v(t))$ and $g(v(\gamma(t)))$ are continuous vector-value activation functions, and $v(t)$ and $v(\gamma(t))$ are the current state and piecewise argument state, respectively.

For the case that $G(v(t))=0$ and $\gamma(t)=t$, NPRNN (1) becomes the following RNN model:

$$
\left\{\begin{array}{l}
\dot{r}(t)=-A r(t)+B f(r(t))+C g(r(t)), \\
r\left(t_{0}\right)=r_{0} \in \mathbb{R}^{n}
\end{array}\right.
$$

Definition 1. For any $t_{0} \in \mathbb{R}^{+}$and $r_{0} \in \mathbb{R}^{n}$, if there are $\alpha, \beta>0$, such that $\left\|r\left(t, t_{0}, r_{0}\right)\right\| \leq \alpha\left\|r_{0}\right\| \exp \left\{-\beta\left(t-t_{0}\right)\right\}$ holds, and the state $r\left(t, t_{0}, r_{0}\right)$ of (2) can achieve globally exponential stability.

Definition 2. For any $t_{0} \in \mathbb{R}^{+}$and $v_{0} \in \mathbb{R}^{n}$, if there are $\widetilde{\alpha}, \widetilde{\beta}>0$, such that $\left\|v\left(t, t_{0}, v_{0}\right)\right\| \leq \widetilde{\alpha}\left\|v_{0}\right\| \exp \left\{-\widetilde{\beta}\left(t-t_{0}\right)\right\}$ holds, and the state $v\left(t, t_{0}, v_{0}\right)$ of (1) can achieve globally exponential stability.

To deduce the main results, some needed assumptions throughout the paper are given:

(H1) The activation functions $f(\cdot), g(\cdot) \in \mathbb{R}^{n}$, and there are Lipschitz constants $l_{1}$ and $l_{2}>0$ such that

$$
\begin{aligned}
& \|f(\varsigma)-f(\varrho)\| \leq l_{1}\|\varsigma-\varrho\|, \\
& \|g(\varsigma)-g(\varrho)\| \leq l_{2}\|\varsigma-\varrho\|,
\end{aligned}
$$

hold for any $\varsigma, \varrho \in \mathbb{R}^{n}$, where $f(\cdot)$ and $g(\cdot)$ are endowed with the initial values $f(0)=0$ and $g(0)=0$.

(H2) Assume that neutral-type function $G(\cdot)$ satisfies a Lipschitz condition:

$$
\|G(\varsigma)-G(\varrho)\| \leq L\|\varsigma-\varrho\|,
$$

where $\varsigma, \varrho \in \mathbb{R}^{n}$.

(H3) For a real-value sequence $\theta_{k}$, there is a $\theta>0$, which makes $\theta_{k+1}-\theta_{k} \leq \theta$ true for any $k \in \mathbf{N}$.

(H4) Assume that

$$
\begin{aligned}
& \left(L+l_{2} \theta\|C\|\right)+\theta\left[\|A\|+l_{1}\|B\|\right]\left\{\frac{1+L+l_{2} \theta\|C\|}{1-L}\right. \\
& \left.\quad \times \exp \left\{\frac{\theta\left(\|A\|+l_{1}\|B\|\right)}{1-L}\right\}\right\}<1 .
\end{aligned}
$$

(H5) Assume that

$$
\begin{gathered}
\alpha \exp \{-\beta(T-\theta)\}+\left[\frac{L \alpha \exp (-\beta \theta)+L}{1-L}+\frac{2 \alpha l_{2}\|C\|}{\beta(1-L)^{2}}\right] \\
\times \exp \left\{\frac{2 T\left(\|A\|+l_{1}\|B\|\right)}{(1-L)}+\frac{2 T\left[(3-L) l_{2}\|C\|\right]}{(1-L)^{2}}\right\}<1 .
\end{gathered}
$$

Remark 1. For the sake of convenience, some symbol descriptions are listed as follows:

$$
\begin{aligned}
\varpi_{1} & =\frac{1+L+l_{2} \theta\|C\|}{1-L}, \\
\varpi_{2} & =\frac{\left[(1+|\sigma|)\|A\|+(1+|\lambda|) l_{1}\|B\|\right]}{1-L}, \\
\varpi_{3} & =\frac{\left[l_{2}\|C\|(1+\Gamma)+|\sigma|\|A\|+|\lambda| l_{1}\|B\|\right] \alpha / \beta}{1-L}+\frac{L \alpha \exp (-\beta \theta)+L}{1-L}, \\
\Phi_{4} & =\frac{\left[(1+|\sigma|)\|A\|+l_{1}(1+|\lambda|)\|B\|+l_{2}\|C\|(2+\Gamma)\right]}{1-L}, \\
\xi & =\left(L+l_{2} \theta\|C\|\right)+\left[(1+|\sigma|)\|A\|+(1+|\lambda|) l_{1}\|B\|\right] \times \theta\left\{\varpi_{1} \exp \left(\varpi_{2} \theta\right)\right\}, \\
\Gamma & =\frac{1+L}{1-\xi}, \quad \xi<1, \\
T & >\ln \frac{\alpha}{\beta}>0 .
\end{aligned}
$$




\section{Main Results}

On the basis of NPRNN (1), in this paper, we mainly explore NPRNN with uncertain connection weights (called parameter-uncertainty NPRNN throughout the text):

$$
\left\{\begin{array}{l}
d[u(t)-G(u(t))]=[-(1+\sigma) A u(t)+(1+\lambda) B f(u(t))+C g(u(\gamma(t)))] \mathrm{d} t \\
u\left(t_{0}\right)=u_{0} \in \mathbb{R}^{n}
\end{array}\right.
$$

where $\sigma$ and $\lambda \in \mathbf{R}$ are the extra interference intensity of connection weight matrices $A$ and $B$, corresponding to the state $u(t)$ and activation function $f(u(t))$.

Then, the following auxiliary Lemma 1 aims to clarify the relationship between piecewise argument state $u(\gamma(t))$ and current state $u(t)$.

Lemma 1. Denote that $u(t)=\left(u_{1}(t), \ldots, u_{n}(t)\right)^{T}$ is a solution of (2), and (H1)-(H4) hold; then, the following inequality,

$$
\|u(\gamma(t))\| \leq \Gamma\|u(t)\|
$$

holds, where

$$
\begin{aligned}
\varpi_{1} & =\frac{1+L+l_{2} \theta\|C\|}{1-L}, \\
\varpi_{2} & =\frac{\left[(1+|\sigma|)\|A\|+(1+|\lambda|) l_{1}\|B\|\right]}{1-L}, \\
\Gamma & =\frac{1+L}{1-\xi}, \quad \xi<1, \\
\xi & =\left(L+l_{2} \theta\|C\|\right)+\left[(1+|\sigma|)\|A\|+(1+|\lambda|) l_{1}\|B\|\right] \times \theta\left\{\varpi_{1} \exp \left(\varpi_{2} \theta\right)\right\} .
\end{aligned}
$$

Proof. Fix $k \in \mathbf{N}$, and for $\gamma(t)=\eta_{k}$ and $t \in\left[\theta_{k}, \theta_{k+1}\right)$, $t \in \mathbb{R}^{+}$, we have

$$
\begin{aligned}
& u(t)-G(u(t))-\left[u\left(\eta_{k}\right)-G\left(u\left(\eta_{k}\right)\right)\right] \\
& =\int_{\eta_{k}}^{t}\left[-(1+\sigma) A u(s)+(1+\lambda) B f(u(s))+C g\left(u\left(\eta_{k}\right)\right)\right] \mathrm{d} s .
\end{aligned}
$$

Applying the norm inequality on both sides of (11) and in accordance with (H1), we obtain

$$
\begin{aligned}
& \| u(t)-u\left(\eta_{k}\right)\|-\| G\left(u\left(\eta_{k}\right)\right)-G(u(t)) \| \\
& \leq(1+|\sigma|)\|A\| \int_{\eta_{k}}^{t}\|u(s)\| \mathrm{d} s+(1+|\lambda|)\|B\| \\
& \times \int_{\eta_{k}}^{t}\|f(u(s))\| \mathrm{d} s+\|C\| \int_{\eta_{k}}^{t}\left\|g\left(u\left(\eta_{k}\right)\right)\right\| \mathrm{d} s \\
& \leq(1+|\sigma|)\|A\| \int_{\eta_{k}}^{t}\|u(s)\| \mathrm{d} s+(1+|\lambda|)\|B\| \\
& \quad \times \int_{\eta_{k}}^{t} l_{1}\|u(s)\| \mathrm{d} s+\|C\| \int_{\eta_{k}}^{t} l_{2}\left\|u\left(\eta_{k}\right)\right\| \mathrm{d} s \\
& \leq(1+|\sigma|)\|A\| \int_{\eta_{k}}^{t}\|u(s)\| \mathrm{d} s+\left[(1+|\lambda|) l_{1}\|B\|\right] \\
& \quad \times \int_{\eta_{k}}^{t}\|u(s)\| \mathrm{d} s+l_{2}\|C\| \int_{\eta_{k}}^{t}\left\|u\left(\eta_{k}\right)\right\| \mathrm{d} s .
\end{aligned}
$$

In terms of (H2) and the norm inequality, we derive

$$
\begin{aligned}
\|u(t)\| \leq & \left\|u\left(\eta_{k}\right)\right\|+L\left\|u\left(\eta_{k}\right)\right\|+L\|u(t)\| \\
& +\left[(1+|\sigma|)\|A\|+(1+|\lambda|) l_{1}\|B\|\right] \int_{\eta_{k}}^{t}\|u(s)\| \mathrm{d} s \\
& +l_{2}\|C\| \int_{\eta_{k}}^{t}\left\|u\left(\eta_{k}\right)\right\| \mathrm{d} s \\
\leq & L\|u(t)\|+\left(1+L+l_{2} \theta\|C\|\right)\left\|u\left(\eta_{k}\right)\right\| \\
& +\left[(1+|\sigma|)\|A\|+(1+|\lambda|) \mid l_{1}\|B\|\right] \int_{\eta_{k}}^{t}\|u(s)\| \mathrm{d} s .
\end{aligned}
$$

Directly, for $L \in(0,1)$, we have

$$
\begin{aligned}
\|u(t)\| \leq & \frac{1+L+l_{2} \theta\|C\|}{1-L}\left\|u\left(\eta_{k}\right)\right\| \\
& +\frac{\left[(1+|\sigma|)\|A\|+(1+|\lambda|) l_{1}\|B\|\right]}{1-L} \int_{\eta_{k}}^{t}\|u(s)\| \mathrm{d} s \\
\leq & \oplus_{1}\left\|u\left(\eta_{k}\right)\right\|+\omega_{2} \int_{\eta_{k}}^{t}\|u(s)\| \mathrm{d} s,
\end{aligned}
$$

where 


$$
\begin{aligned}
& \varpi_{1}=\frac{1+L+l_{2} \theta\|C\|}{1-L}, \\
& \varpi_{2}=\frac{\left[(1+|\sigma|)\|A\|+(1+|\lambda|) l_{1}\|B\|\right]}{1-L} .
\end{aligned}
$$

According to Gronwall-Bellman lemma, it follows that

$$
\|u(t)\| \leq \Phi_{1} \exp \left\{\Phi_{2} \theta\right\}\left\|u\left(\eta_{k}\right)\right\| .
$$

Moreover, for $t \in\left[\theta_{k}, \theta_{k+1}\right)$, we obtain

$$
\begin{aligned}
& G\left(u\left(\eta_{k}\right)\right)-G(u(t)) \\
& =u\left(\eta_{k}\right)-u(t)+\int_{\eta_{k}}^{t}\left[-(1+\sigma) A u(s)+(1+\lambda) B f(u(s))+C g\left(u\left(\eta_{k}\right)\right)\right] \mathrm{d} s
\end{aligned}
$$

In combination with (H1)-(H3) and (16), similarly, we obtain

$$
\begin{aligned}
\left\|u\left(\eta_{k}\right)\right\| \leq & \|u(t)\|+L\left\|u\left(\eta_{k}\right)\right\|+L\|u(t)\|+[(1+|\sigma|)\|A\| \\
& \left.+(1+|\lambda|) l_{1}\|B\|\right] \int_{\eta_{k}}^{t}\|u(s)\| \mathrm{d} s \\
& +l_{2}\|C\| \int_{\eta_{k}}^{t}\left\|u\left(\eta_{k}\right)\right\| \mathrm{d} s \\
\leq & (1+L)\|u(t)\|+L\left\|u\left(\eta_{k}\right)\right\|+[(1+|\sigma|)\|A\| \\
& \left.+(1+|\lambda|) l_{1}\|B\|\right] \theta\left\{\varpi_{1} \exp \left\{\varpi_{2} \theta\right\}\right\}\left\|u\left(\eta_{k}\right)\right\| \\
& +l_{2}\|C\| \int_{\eta_{k}}^{t}\left\|u\left(\eta_{k}\right)\right\| \mathrm{d} s \\
\leq & (1+L)\|u(t)\|+\left\{\left(L+l_{2} \theta\|C\|\right)+[(1+|\sigma|)\|A\|\right. \\
& \left.\left.+(1+|\lambda|) l_{1}\|B\|\right] \theta\left\{\varpi_{1} \exp \left(\varpi_{2} \theta\right)\right\}\right\}\left\|u\left(\eta_{k}\right)\right\| \\
\leq & (1+L)\|u(t)\|+\xi\left\|u\left(\eta_{k}\right)\right\|,
\end{aligned}
$$

where

$$
\begin{aligned}
\xi= & \left(L+l_{2} \theta\|C\|\right)+\left[(1+|\sigma|)\|A\|+(1+|\lambda|) l_{1}\|B\|\right] \\
& \times \theta\left\{\varpi_{1} \exp \left(\varpi_{2} \theta\right)\right\} .
\end{aligned}
$$

Therefore, by (18) and (H4), for $\eta_{k}=\gamma(t)$ and $k \in \mathbf{N}$, we can obtain

$$
\begin{aligned}
\|u(\gamma(t))\| & \leq \frac{1+L}{1-\xi}\|u(t)\| \\
& =: \Gamma\|u(t)\|,
\end{aligned}
$$

where

$$
\Gamma=\frac{1+L}{1-\xi}, \quad \xi<1 .
$$

Theorem 1. If (H1)-(H5) hold, (2) achieves globally exponential stability. Then, the conditions for achieving the RoGES of NPRNN can be given by the following (a) and (b): (a) Let $\bar{L}$ be the upper bound of neutral term compressibility coefficient; then, $L<\bar{L}$ and $\bar{L} \in(0,1)$, and $\bar{L}$ is given by the following transcendental equation:

$$
\begin{aligned}
& \alpha \exp (-\beta T)+\frac{(\bar{L} \alpha+\bar{L})(1-\bar{L}) \beta+2 \alpha l_{2}\|C\|}{\beta(1-\bar{L})^{2}} \\
& \quad \times \exp \left\{\frac{2 T\left[(1-\bar{L})\left(\|A\|+l_{1}\|B\|\right)+(3-\bar{L}) l_{2}\|C\|\right]}{(1-\bar{L})^{2}}\right\}<1 .
\end{aligned}
$$

(b) Let $\theta_{3}$ be the upper bound of piecewise constant arguments, where $\theta_{3}$ is given by

$$
\theta<\theta_{3}=\min \left\{\frac{T}{2}, \theta_{1}, \theta_{2}\right\},
$$

where $\theta_{1}$ and $\theta_{2}$ are the upper bounds satisfying (H4) and (H5), respectively, $T>(\ln \alpha) / \beta>0$.

Furthermore, if the real selected values $L<\bar{L}$ and $\theta<\theta_{3}$ hold by (22) and (23), then the allowed intensity of $(\sigma, \lambda)$ to achieve the RoGES of parameter-uncertainty NPRNN (8) should be in the inner of the following enclosed curve:

$$
\alpha \exp (-\beta(T-\theta))+\varpi_{3} \exp \left\{2 T \varpi_{4}\right\}=1,
$$

where

$$
\begin{aligned}
\varpi_{3}= & \frac{L \alpha \exp (-\beta \theta)+L}{1-L} \\
& +\frac{\left[l_{2}\|C\|(1+\Gamma)+|\sigma|\|A\|+|\lambda| l_{1}\|B\|\right] \alpha / \beta}{1-L},
\end{aligned}
$$

$$
\varpi_{4}=\frac{\left[(1+|\sigma|)\|A\|+l_{1}(1+|\lambda|)\|B\|+l_{2}\|C\|(2+\Gamma)\right]}{1-L},
$$

and the other parameters are the same as Lemma 1. Besides, the boundary value of $|\sigma|$ is expressed as $|\sigma|_{\text {sup }}$, and $|\lambda|$ is expressed as $|\lambda|_{\text {sup }}$ hereinbelow.

Proof. From (2) and (8), for $t \geq t_{0}$, we obtain 


$$
\begin{aligned}
& u(t)-r(t)=G(u(t))-G\left(u\left(t_{0}\right)\right) \\
& +\int_{t_{0}}^{t}\{-A[u(s)-r(s)]+B[f(u(s))-f(r(s))] \\
& \quad+C[g(u(\gamma(s)))-g(u(s))+g(u(s))-g(r(s))] \\
& \quad-\sigma A[u(s)-r(s)+r(s)]
\end{aligned}
$$$$
+\lambda B[f(u(s))-f(r(s))+f(r(s))]\} \mathrm{d} s .
$$

$$
\begin{aligned}
\|u(t)-r(t)\| \leq & \left\|G(u(t))-G\left(u\left(t_{0}\right)\right)\right\|+\left[\|A\|+l_{1}\|B\|+l_{2}\|C\|\right. \\
& \left.+|\sigma|\|A\|+|\lambda| l_{1}\|B\|\right] \int_{t_{0}}^{t}\|u(s)-r(s)\| \mathrm{d} s \\
& +\|C\| \int_{t_{0}}^{t}\|g(u(\gamma(s)))-g(u(s))\| \mathrm{d} s \\
& +\left(|\sigma|\|A\|+|\lambda| l_{1}\|B\|\right) \int_{t_{0}}^{t}\|r(s)\| \mathrm{d} s \\
\leq & L\|u(t)\|+L\left\|u\left(t_{0}\right)\right\|+\left[(1+|\sigma|)\|A\|+l_{1}(1+|\lambda|)\|B\|\right. \\
& \left.+l_{2}\|C\|\right] \int_{t_{0}}^{t}\|u(s)-r(s)\| \mathrm{d} s \\
& +\|C\| l_{2} \int_{t_{0}}^{t}(\|u(\gamma(s))\|+\|u(s)\|) \mathrm{d} s \\
& +\left(|\sigma|\|A\|+|\lambda| l_{1}\|B\|\right) \int_{t_{0}}^{t}\|r(s)\| \mathrm{d} s \\
& +\left(|\sigma|\|A\|+|\lambda| l_{1}\|B\|\right) \int_{t_{0}}^{t}\|r(s)\| \mathrm{d} s \\
& +l_{2}\|C\|(t)-r(t)\|+L\| r(t)\|+L\| u\left(t_{0}\right) \|+\left[(1+|\sigma|)\|A\|+l_{1}(1+|\lambda|)\|B\|\right. \\
& +\|(s)-r(s)\| \mathrm{d} s \\
& \\
& \\
& \\
& \\
& \\
&
\end{aligned}
$$

In accordance with Definition 1 and the exponential stability of (2), $L \in(0,1)$, when $t \in\left[t_{0}-\theta, t_{0}+\theta\right]$, further we derive 


$$
\begin{aligned}
\|u(t)-r(t)\| \leq & \frac{L}{1-L} \alpha\left\|u_{0}\right\| \exp (-\beta \theta)+\frac{L}{1-L}\left\|u_{0}\right\| \\
& +\frac{\left[(1+|\sigma|)\|A\|+l_{1}(1+|\lambda|)\|B\|+l_{2}\|C\|(2+\Gamma)\right]}{1-L} \\
& \times \int_{t_{0}}^{t}\|u(s)-r(s)\| \mathrm{d} s \\
& +\frac{l_{2}\|C\|(1+\Gamma)+|\sigma|\|A\|+|\lambda| l_{1}\|B\|}{1-L} \\
& \times \int_{t_{0}}^{t} \alpha\left\|r_{0}\right\| \exp \left(-\beta\left(s-t_{0}\right)\right) \mathrm{d} s \\
\leq & \left\{\frac{L \alpha \exp (-\beta \theta)+L}{1-L}\right. \\
& \left.+\frac{\left[l_{2}\|C\|(1+\Gamma)+|\sigma|\|A\|+|\lambda| l_{1}\|B\|\right] \alpha / \beta}{1-L}\right\}\left\|u_{0}\right\| \\
& +\frac{\left[(1+|\sigma|)\|A\|+l_{1}(1+|\lambda|)\|B\|+l_{2}\|C\|(2+\Gamma)\right]}{1-L} \\
& \times \int_{t_{0}}^{t}\|u(s)-r(s)\| \mathrm{d} s \\
\leq & \left\|u_{0}\right\|+\Phi_{4} \int_{t_{0}}^{t}\|u(s)-r(s)\| \mathrm{d} s,
\end{aligned}
$$

where

$$
\begin{aligned}
& \varpi_{3}=\frac{L \alpha \exp (-\beta \theta)+L}{1-L}+\frac{\left[l_{2}\|C\|(1+\Gamma)+|\sigma|\|A\|+|\lambda| l_{1}\|B\|\right] \alpha / \beta}{1-L}, \\
& \varpi_{4}=\frac{\left[(1+|\sigma|)\|A\|+l_{1}(1+|\lambda|)\|B\|+l_{2}\|C\|(2+\Gamma)\right]}{1-L} .
\end{aligned}
$$

By virtue of Gronwall inequality, for $t_{0}+\theta \leq t \leq t_{0}+2 T$, we have

$$
\begin{aligned}
\|u(t)-r(t)\| & \leq \varpi_{3}\left\|u_{0}\right\| \exp \left\{\varpi_{4}\left(t-t_{0}\right)\right\} \\
& \leq \varpi_{3} \exp \left\{2 T \varpi_{4}\right\} \sup _{t \in\left[t_{0}-\theta, t_{0}+\theta\right]}\|u(t)\| .
\end{aligned}
$$

Note that $\theta<(T / 2)$, when $t_{0}-\theta+T \leq t_{0}-\theta+2 T$, by the exponential stability of (2), we further obtain

$$
\begin{aligned}
\|u(t)\| \leq & \|r(t)\|+\|u(t)-r(t)\| \\
\leq & {\left[\alpha \exp (-\beta(T-\theta))+\Phi_{3} \exp \left\{2 T \Phi_{4}\right\}\right] } \\
& \times \sup _{t_{0}-\theta \leq t_{0}-\theta+T}\|u(t)\| \\
= & : \widetilde{C} \sup _{t_{0}-\theta \leq t_{0}-\theta+T}\|u(t)\|,
\end{aligned}
$$

where $\widetilde{C}=\alpha \exp (-\beta(T-\theta))+\varpi_{3} \exp \left\{2 T \varpi_{4}\right\}$. Then, if we define

$$
F(\Gamma(\theta), L)=\alpha \exp \{-\beta(T-\theta)\}+\varpi_{3} \exp \left\{2 T \varpi_{4}\right\},
$$

obviously, there is

$$
F(\Gamma(\theta), L) \leq 1 .
$$

Firstly, by (H4), denote $\theta_{1}$ as the unique maximal positive solution satisfying $\left.\xi(\theta)\right|_{\sigma, \lambda=0}<1$, that is,

$$
\begin{aligned}
& \left(L+l_{2} \theta\|C\|\right)+\theta\left[\|A\|+l_{1}\|B\|\right]\left\{\frac{1+L+l_{2} \theta\|C\|}{1-L}\right. \\
& \left.\exp \left\{\frac{\theta\left(\|A\|+l_{1}\|B\|\right)}{1-L}\right\}\right\}<1,
\end{aligned}
$$

so

$$
\Gamma(\theta)=\frac{1+L}{1-\xi(\theta)} \in\left(\frac{1+L}{1-L}, \infty\right), \quad L \in(0,1),
$$

holds for any $\theta \in\left(0, \theta_{1}\right)$. According to (32) and (H5), it derives

$$
\begin{aligned}
\left.F\left(\frac{1+L}{1-L}, L\right)\right|_{\lambda, \sigma=0}= & \alpha \exp \{-\beta(T-\theta)\}+\left[\frac{L \alpha \exp (-\beta \theta)+L}{1-L}+\frac{2 \alpha l_{2}\|C\|}{\beta(1-L)^{2}}\right] \\
& \times \exp \left\{\frac{2 T\left(\|A\|+l_{1}\|B\|\right)}{(1-L)}+\frac{2 T\left[(3-L) l_{2}\|C\|\right]}{(1-L)^{2}}\right\}<1,
\end{aligned}
$$

$$
F(\infty, L)>1 .
$$

Thus, by the monotonicity of $F(\Gamma(\theta), L)$, there exists some $\widehat{\Gamma}(\theta) \in(((1+l) /(1-L)), \infty)$, which makes $F(\Gamma(\theta)$, $L)\left.\right|_{\lambda, \sigma=0}=1$ true. Then, if we denote $\theta_{2}$ as the upper bound satisfying (36) and select

$$
\theta_{3}=\min \left\{\frac{T}{2}, \theta_{1}, \theta_{2}\right\},
$$

hence, the upper bound of the piecewise arguments unaffected by the intensity of uncertain parameters is given. 
Besides, since $\partial(\Gamma(\theta)) / \partial \theta>0$ and $\partial(F(\Gamma(\theta)), L) / \partial \theta>0$, the upper bound of neutral terms can be given by

$$
\begin{aligned}
\left.F\left(\frac{1+L}{1-L}, L\right)\right|_{\theta, \lambda, \sigma=0}= & \alpha \exp (-\beta T)+\frac{(L \alpha+L)(1-L) \beta+2 \alpha l_{2}\|C\|}{\beta(1-L)^{2}} \\
& \times \exp \left\{\frac{2 T\left[(1-L)\left(\|A\|+l_{1}\|B\|\right)+(3-L) l_{2}\|C\|\right]}{(1-L)^{2}}\right\}<1 .
\end{aligned}
$$

Finally, if we select the exact $L$ and $\theta$ which stabilize NPRNN (1), the intensity boundary of dual parameters $\sigma$ and $\lambda$ to stabilize the new parameter-uncertainty NPRNN (8) will be obtained by the following transcendental equation in accordance with the above $L$ and $\theta$ :

$$
\alpha \exp (-\beta(T-\theta))+\varpi_{3} \exp \left\{2 T \varpi_{4}\right\}=1,
$$

and some corresponding symbolic descriptions of (40) are shown in Remark 1.
Accordingly, selecting $\Lambda=-\ln \widetilde{C} / T$, where $\Lambda \geq 0$, and from (31), we can obtain

$$
\begin{aligned}
& \sup _{t_{0}-\theta+T \leq t \leq t_{0}-\theta+2 T}\left\|u\left(t, t_{0}, u_{0}\right)\right\| \\
& \leq \exp (-\Lambda T) \sup _{t_{0}-\theta \leq t \leq t_{0}-\theta+T}\left\|u\left(t, t_{0}, u_{0}\right)\right\| .
\end{aligned}
$$

Subsequently, for any positive integer $q \in \underline{n}$, when $t \geq t_{0}-\theta+(q-1) T$, by the continuity of the solutions of the system [13], invoking (41), further we have

$$
\begin{aligned}
& \sup _{t_{0}-\theta+q T \leq t \leq t_{0}-\theta+(q+1) T}\left\|u\left(t ; t_{0}, u_{0}\right)\right\| \\
& =\sup _{t_{0}-\theta+(q-1) T+T \leq t \leq t_{0}-\theta+q T+T} \| u\left(t ; t_{0}-\theta+(q-1) T,\right. \\
& \left.u\left(t_{0}-\theta+(q-1) T ; t_{0}, u_{0}\right)\right) \| \\
& \quad \leq \exp (-\Lambda T) \sup _{t_{0}-\theta+(q-1) T \leq t \leq t_{0}-\theta+q T}\left\|u\left(t ; t_{0}, u_{0}\right)\right\| \\
& \quad \leq \exp (-\Lambda q T) \sup _{t_{0}-\theta \leq t \leq t_{0}-\theta+T}\left\|u\left(t ; t_{0}, u_{0}\right)\right\| \\
& =C \exp (-\Lambda q T),
\end{aligned}
$$

where $C=\sup _{t_{0}-\theta \leq t \leq t_{0}-\theta+T}\left\|u\left(t, t_{0}, u_{0}\right)\right\|$. Hence, when $t_{0}-\theta+q T \leq t \leq t_{0}-\theta+(q+1) T$, for any integer $q \in \underline{n}$, we have

$$
\begin{aligned}
\|u(t)\| & \leq C \exp (-\Lambda q T) \\
& \leq C \exp \left\{\Lambda\left[-\left(t-t_{0}\right)+(T-\theta)\right]\right\} \\
& \leq C \exp (\Lambda T) \exp \left(-\Lambda\left(t-t_{0}\right)\right) .
\end{aligned}
$$

In this way, (8) achieves globally exponential stability.

Corollary 1. Obviously, the allowed intensity of $(\sigma, \lambda)$ to achieve the RoGES of parameter-uncertainty NPRNN (8) is derived in Theorem 1. In fact, Theorem 1 is also applicable for the following models (44) and (45):

$$
\begin{aligned}
& \left\{\begin{array}{l}
d[u(t)-G(u(t))]=[-(1+\sigma) A u(t)+B f(u(t))+C g(u(\gamma(t)))] \mathrm{d} t, \\
u\left(t_{0}\right)=u_{0} \in \mathbb{R}^{n},
\end{array}\right. \\
& \left\{\begin{array}{l}
d[u(t)-G(u(t))]=[-A u(t)+(1+\lambda) B f(u(t))+C g(y(\gamma(t)))] \mathrm{d} t, \\
u\left(t_{0}\right)=u_{0} \in \mathbb{R}^{n},
\end{array}\right.
\end{aligned}
$$

corresponding to the cases $\lambda=0$ and $\sigma=0$ for parameteruncertainty NPRNN (8), respectively.
Proof. Similarly, if we set $\lambda=0$ and $\sigma=0$ for Theorem 1, respectively, the sufficient conditions to ensure the globally 
exponential stability of (44) and (45) can be given promptly.

Remark 2. It is not easy to directly handle (24) (i.e., (40)), a transcendental equation with four variables in Theorem 1. Hence, we adopt a special method: fix $\theta$ and $L$ which can maintain the globally exponential stability of NPRNN (1), and then, in combination with some known parameters $\alpha, \beta$, $l_{1}, l_{2}$, and $T,(24)$ becomes an implicit transcendental equation with only two variables $|\sigma|$ and $|\lambda|$. Transcendental equation (24) is a key step to obtain the enclosed curve such as the one in Figure 1, which is also the core work of this paper.

Remark 3. The order of the calculation in this paper is as follows. At first, some parameters $\alpha, \beta, l_{1}, l_{2}$, and $T$ are given in advance, and the upper bound of the neutral terms $(\bar{L})$ can be derived by (22). Next, we fix an exact $L<\bar{L}$ which can ensure the exponential stability of NPRNN (1), and then, the supremum of the piecewise arguments $\left(\theta_{3}\right)$ is obtained by (23). Furthermore, in accordance with the appropriately selected values of $L$ and $\theta$ (i.e., $L<\bar{L}$ and $\theta<\theta_{3}$ ) and equation (24), we can obtain the bounds of $\sigma$ and $\lambda$ which can guarantee the RoGES of parameter-uncertainty NPRNN (8) by MATLAB. The robustness of the system means that if the

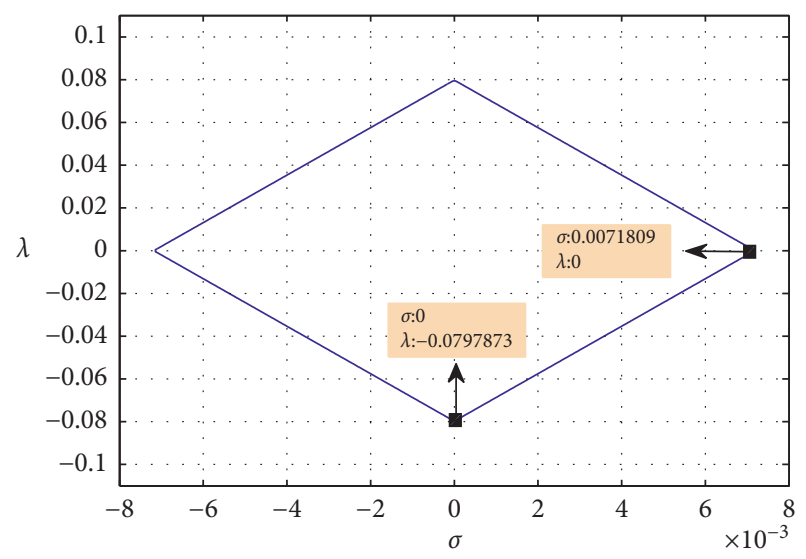

Figure 1: Stable enclosed curve of $\sigma$ and $\lambda$ in system (48).

original NPRNN (1) is stable, the perturbed parameteruncertainty NPRNN (8) can still remain stable as long as the selected values of $\sigma$ and $\lambda$ are included inside the closed curve given by equation (24).

\section{Illustrative Example}

Example 1. For a two-state RNN,

$$
\begin{aligned}
\left(\begin{array}{c}
\dot{r}_{1}(t) \\
\dot{r}_{2}(t)
\end{array}\right)= & -\left(\begin{array}{ll}
1 & 0 \\
0 & 1
\end{array}\right)\left(\begin{array}{l}
r_{1}(t) \\
r_{2}(t)
\end{array}\right)+\left(\begin{array}{cc}
0.09 & 0 \\
0 & 0.09
\end{array}\right)\left(\begin{array}{c}
\tanh \left(r_{1}(t)\right) \\
\tanh \left(r_{2}(t)\right)
\end{array}\right) \\
& +\left(\begin{array}{cc}
0.1 & 0.1 \\
0.1 & 0.1
\end{array}\right)\left(\begin{array}{l}
\sin \left(\frac{r_{1}(t)}{2}\right) \\
\sin \left(\frac{r_{2}(t)}{2}\right)
\end{array}\right) .
\end{aligned}
$$

Equation (46) can achieve globally exponential stability with $\alpha=1$ and $\beta=0.6$. According to Theorem 1 , let $T=0.5>\ln 1 / 0.6=0$. From $(\mathrm{H} 1)$, let $l_{1}=1$ and $l_{2}=0.5$. Besides, other parameters $\|A\|=1,\|B\|=0.09$, and $\|C\|=$
0.02 can also be listed; then, the stable state trajectory can be seen in Figure 2.

When the neutral terms and piecewise arguments are attached to RNN (46), (46) can be written as NPRNN (47):

$$
\begin{aligned}
\frac{\mathrm{d}}{\mathrm{d} t}\left(\begin{array}{c}
v_{1}(t)+L \sin \left(v_{1}(t)\right) \\
v_{2}(t)+L \sin \left(v_{2}(t)\right)
\end{array}\right)= & -\left(\begin{array}{ll}
1 & 0 \\
0 & 1
\end{array}\right)\left(\begin{array}{l}
v_{1}(t) \\
v_{2}(t)
\end{array}\right)+\left(\begin{array}{cc}
0.09 & 0 \\
0 & 0.09
\end{array}\right)\left(\begin{array}{c}
\tanh \left(v_{1}(t)\right) \\
\tanh \left(v_{2}(t)\right)
\end{array}\right) \\
& +\left(\begin{array}{ll}
0.1 & 0.1 \\
0.1 & 0.1
\end{array}\right)\left(\begin{array}{c}
\sin \left(\frac{v_{1}(\gamma(t))}{2}\right) \\
\sin \left(\frac{v_{2}(\gamma(t))}{2}\right)
\end{array}\right),
\end{aligned}
$$




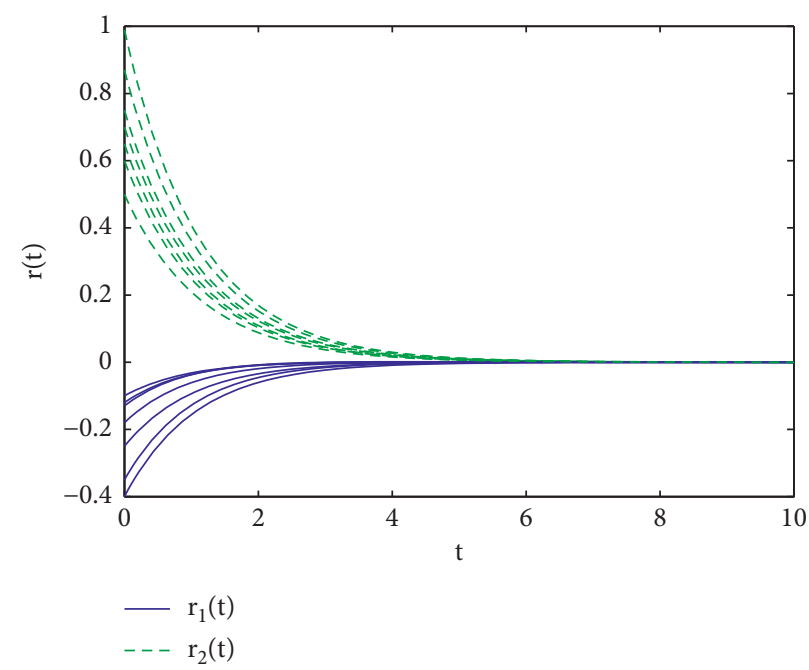

FIgURE 2: Stable state trajectories of $r_{1}(t)$ and $r_{2}(t)$ in (46).

where $\left\{\theta_{k}\right\}=\{k / 10\}$ and $\left\{\eta_{k}\right\}=\{2 k+1 / 20\}, \quad k \in \mathbf{N}$. The piecewise constant argument function $\gamma(t)=\eta_{k}$ when $t \in\left[\theta_{k}, \theta_{k+1}\right), k \in \mathbf{N}$.
Lastly, if we consider additional dual-parameter perturbations for (47), then parameter-uncertainty NPRNN (48) is formulated as

$$
\begin{aligned}
\frac{\mathrm{d}}{\mathrm{d} t}\left(\begin{array}{c}
u_{1}(t)+L \sin \left(u_{1}(t)\right) \\
u_{2}(t)+L \sin \left(u_{2}(t)\right)
\end{array}\right)= & -(1+\sigma)\left(\begin{array}{ll}
1 & 0 \\
0 & 1
\end{array}\right)\left(\begin{array}{c}
u_{1}(t) \\
u_{2}(t)
\end{array}\right)+(1+\lambda)\left(\begin{array}{cc}
0.09 & 0 \\
0 & 0.09
\end{array}\right) \\
& \times\left(\begin{array}{c}
\tanh \left(u_{1}(t)\right) \\
\tanh \left(u_{2}(t)\right)
\end{array}\right)+\left(\begin{array}{ll}
0.1 & 0.1 \\
0.1 & 0.1
\end{array}\right)\left(\begin{array}{c}
\sin \left(\frac{u_{1}(\gamma(t))}{2}\right) \\
\sin \left(\frac{u_{2}(\gamma(t))}{2}\right)
\end{array}\right) .
\end{aligned}
$$

In order to make the process clearer, the following explanations will be divided into two parts to illustrate the RoGES of system (48). On the one hand, we will explain how much the interference intensity of neutral terms and piecewise arguments the system (47) can tolerate to be stable again based on the stable RNN (46) depicted in Figure 2.

Firstly, calculating the following equation by MATLAB,

$$
\exp (-0.3)+\frac{2 L(1-L) \times 0.6+0.02}{0.6 \times(1-L)^{2}} \times \exp \left\{\frac{1.09 \times(1-L)+0.01 \times(3-L)}{(1-L)^{2}}\right\}=1
$$

then the upper bound of $L$ is obtained: $\bar{L}=0.0231$.

Next, if we select $L=0.01<\bar{L}=0.0231$ and substitute $L$, $\alpha, \beta, l_{1}, l_{2}$, and $T$ into (H4) and (H5), we get $\theta_{1}=0.5053$ and $\theta_{2}=0.2004$, respectively. So, the upper bound of $\theta$ is given by the following $\theta_{3}$ :

$$
\begin{aligned}
\theta<\theta_{3} & =\min \left\{\frac{T}{2}, \theta_{1}, \theta_{2}\right\} \\
& =\min \{0.25,0.5053,0.2004\} \\
& =0.2004 .
\end{aligned}
$$

According to Theorem 1, the states $v_{1}(t)$ and $v_{2}(t)$ of (47) will be stable with $L=0.01$ and $\theta=0.1$, which is shown in Figure 3.

On the other hand, we will explain the intensity of connection weights $\sigma$ and $\lambda$ that parameter-uncertainty NPRNN (48) can tolerate based on the stable NPRNN (47) shown in Figure 3.

Therefore, we fix parameters $L=0.01$ and $\theta=0.1$ so as to satisfy the stable conditions in Theorem 1 and be consistent with the parameter setting in Figure 3. Subsequently, the stable region with $(\sigma, \lambda)$ can be solved by a transcendental equation by MATLAB: 


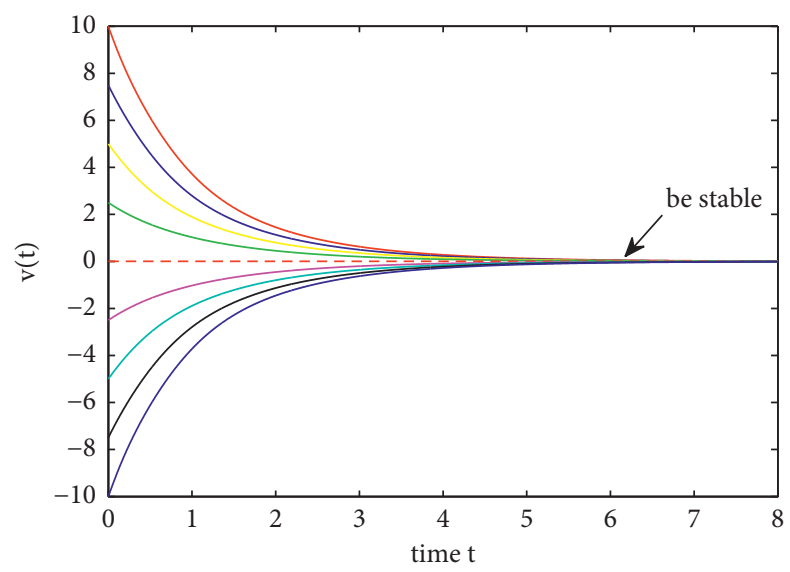

Figure 3: Stability of $v_{1}(t)$ and $v_{2}(t)$ in (47) with $L=0.01$ and $\theta=0.1$.

$$
\begin{aligned}
& \exp (-0.24)+\frac{0.01 \times \exp (-0.06)+0.01}{0.99} \\
& +\frac{[0.01 \times(1+(1.01 /(1-\xi)))+|\sigma|+0.09|\lambda|] \times(1 / 0.6)}{0.99} \\
& \quad \times \exp \left\{\frac{(1+|\sigma|)+0.09(1+|\lambda|)+0.01 \times(2+(1.01 /(1-\xi)))}{0.99}\right\}=1,
\end{aligned}
$$

where

$$
\begin{aligned}
\xi= & 0.011+[(1+|\sigma|)+0.09(1+|\lambda|)] \times 0.1 \\
& \times\left\{\frac{1.011}{0.99} \exp \left\{\frac{0.1 \times[(1+|\sigma|)+0.09(1+|\lambda|)]}{0.99}\right\}\right\} .
\end{aligned}
$$

According to the above implicit transcendental equation, the stable region of $\sigma$ and $\lambda$ is depicted in Figure 1, that is, if the intensity range of $(\sigma, \lambda)$ is in the inner of the enclosed curve in Figure 1, then parameter-uncertainty NPRNN (48) will be stable again based on the stable NPRNN (47). Therefore, some stable and unstable cases are given to verify the RoGES of system (48), such as the stable case (i) and unstable cases (ii)-(v).

(i) If $(\sigma, \lambda)=(0.001,0.01)$, where $|\sigma|=0.001<|\sigma|_{\text {sup }}=$ 0.0071809 and $|\lambda|=0.01<|\lambda|_{\text {sup }}=0.0797873$, according to Figure 1, the stable state trajectories of $u_{1}(t)$ and $u_{2}(t)$ are shown in Figure 4 .

Otherwise, some unstable cases should be provided to illustrate. If the connection weight $\sigma$ exceeds the boundary value $|\sigma|_{\text {sup }}$ or $\lambda$ exceeds the boundary value $|\lambda|_{\text {sup }}$ in Figure 1, parameter-uncertainty NPRNN (48) will be unstable even based on stable NPRNN (47), which can be illustrated in the following:

(ii) If $(\sigma, \lambda)=(0,18)$, where $|\lambda|=18>|\lambda|_{\text {sup }}=0.0797873$, the unstable state trajectories are shown in Figure 5.

(iii) If $(\sigma, \lambda)=(-1.6,0)$, where $|\sigma|=1.6>|\sigma|_{\text {sup }}=$ 0.0071809 , the unstable state trajectories are shown in Figure 6. (iv) If $(\sigma, \lambda)=(0.5,20)$, where $|\sigma|=0.5>|\sigma|_{\text {sup }}=$ 0.0071809 and $|\lambda|=20>|\lambda|_{\text {sup }}=0.0797873$, the unstable state trajectories are shown in Figure 7.

(v) If $(\sigma, \lambda)=(-0.5,20)$, similarly, the unstable state trajectories are shown in Figure 8.

Remark 4. Figure 1 is an enclosed curve about $\sigma$ and $\lambda$ with the boundary value $|\sigma|_{\text {sup }}$ and $|\lambda|_{\text {sup }}$. Figure 1 is depicted by (24); more details of the calculation process of (24) can be seen in Remark 2. According to Remark 2, we transform (24) from a four-variable equation to a two-variable equation only related to $|\sigma|$ and $|\lambda|$. By virtue of computer software MATLAB, an enclosed region of $\sigma$ and $\lambda$, and the boundary values $|\sigma|_{\text {sup }}=0.0071809$ and $|\lambda|_{\text {sup }}=0.0797873$ are all directly exhibited in Figure 1 to demonstrate the RoGES of parameter-uncertainty NPRNN (48). If we select $|\sigma|<|\sigma|_{\text {sup }}$ and $|\lambda|<|\lambda|_{\text {sup }}$, system (48) will be stable again based on stable Figure 3. Or else, if $|\sigma|>|\sigma|_{\text {sup }}$ or $|\lambda|>|\lambda|_{\text {sup }}$ holds, system (48) will lose its stability even on the basis of stable Figure 3.

Remark 5. Figures 1-8 systematically and intuitively prove the robustness of the system (48). From Theorem 1, the supremum of neutral terms $\bar{L}$ is derived by (22), and the supremum of piecewise arguments $\theta_{3}$ is derived by (23), and the boundary of uncertain-parameter intensity is characterized by (24). Figures 1-8 are obtained as follows. (1) Fix some necessary parameters $\alpha, \beta, l_{1}, l_{2}$, and $T$; an initial globally exponentially stable RNN (46) is given by Figure 2. (2) According to $\bar{L}$ and $\theta_{3}$ settled by (22) and (23), here we select $L=0.01<\bar{L}=0.0231$ and 


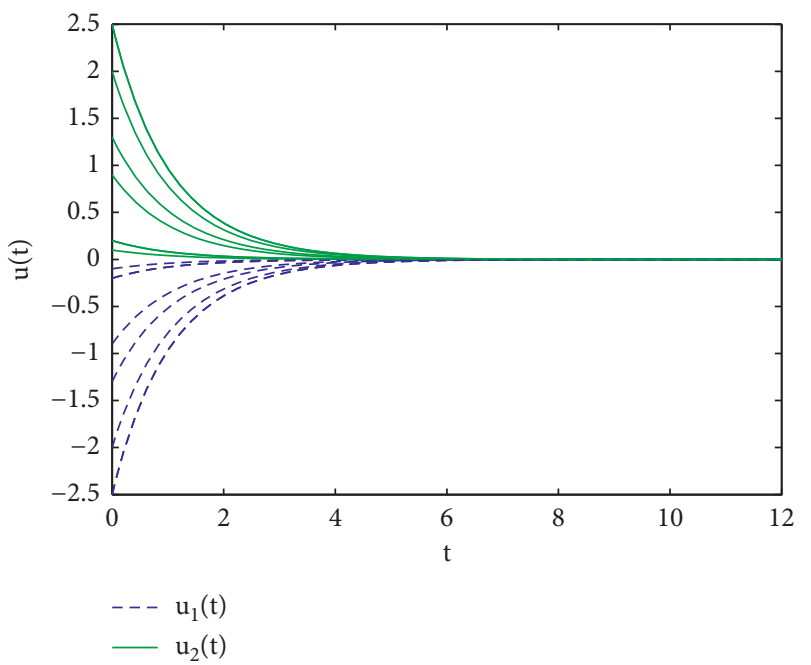

FIgURE 4: Stability of $u_{1}(t)$ and $u_{2}(t)$ in (48) with $(\sigma, \lambda)=(0.001,0.01)$.

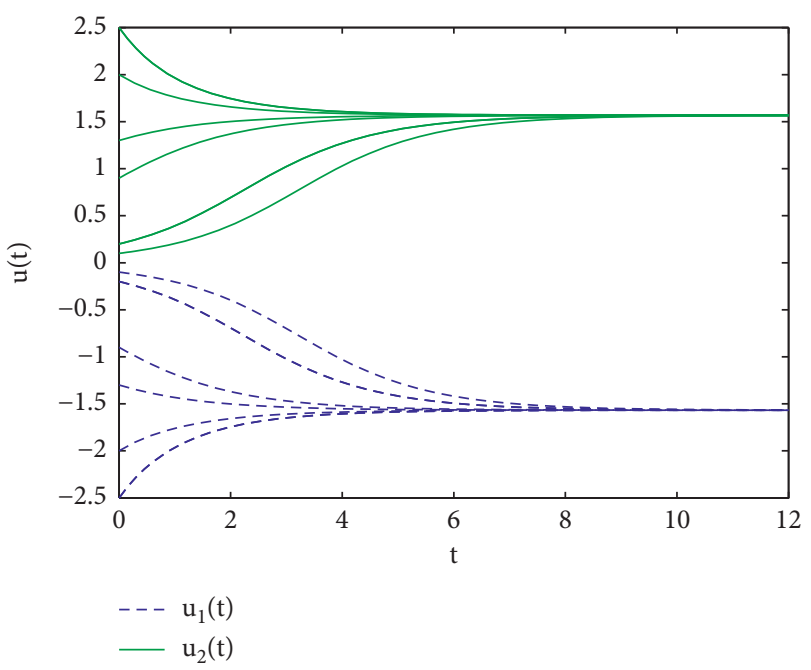

FIGURE 5: Instability of $u_{1}(t)$ and $u_{2}(t)$ in (48) with $(\sigma, \lambda)=(0,18)$.

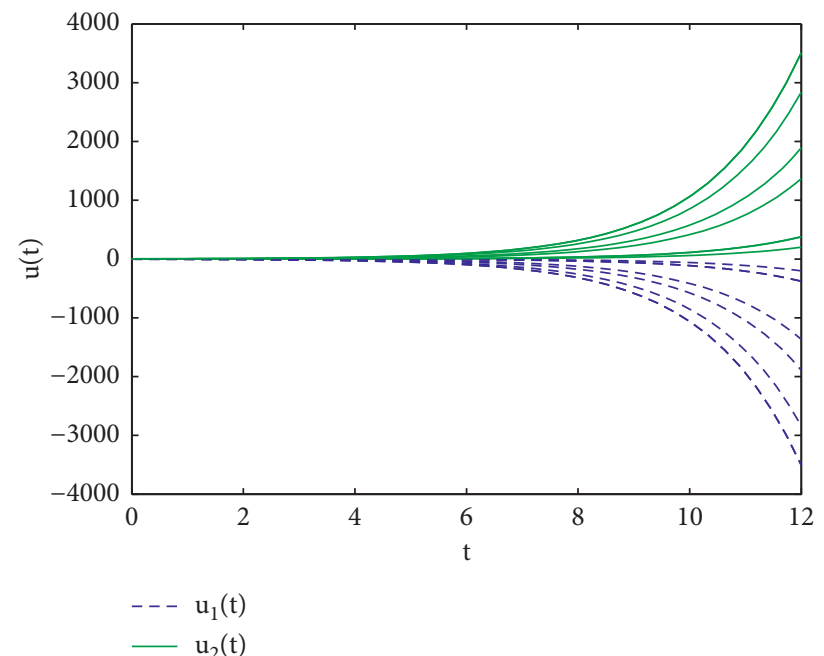

FIGURE 6: Instability of $u_{1}(t)$ and $u_{2}(t)$ in (48) with $(\sigma, \lambda)=(-1.6,0)$. 


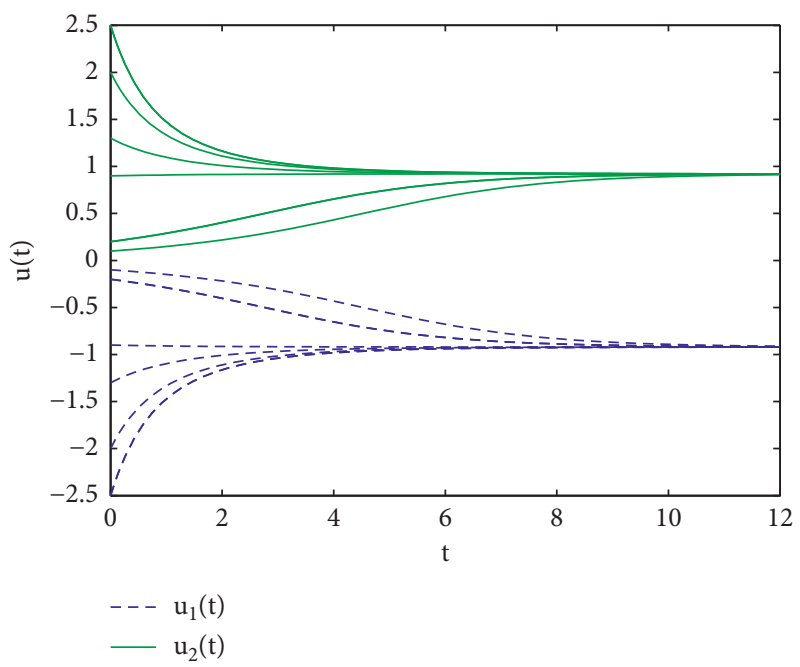

Figure 7: Instability of $u_{1}(t)$ and $u_{2}(t)$ in (48) with $(\sigma, \lambda)=(0.5,20)$.

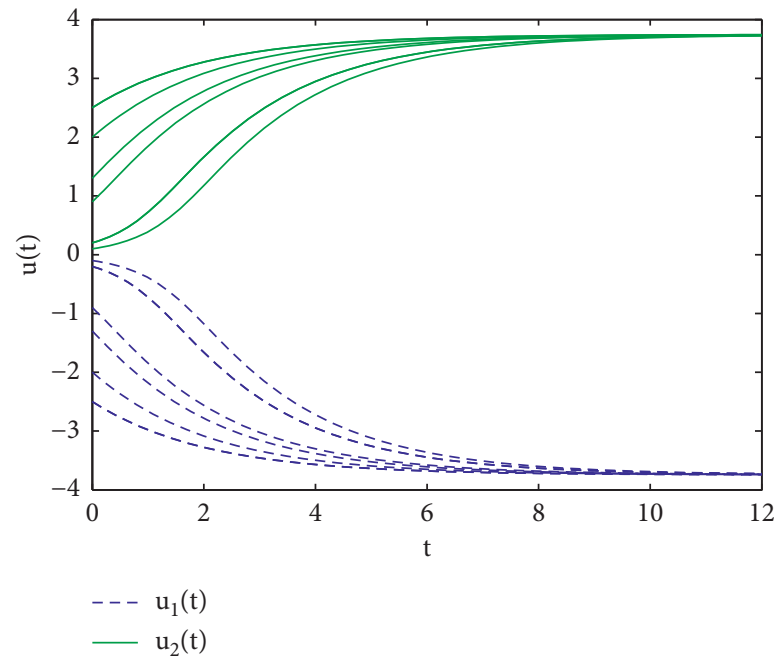

FIGURE 8: Instability of $u_{1}(t)$ and $u_{2}(t)$ in (48) with $(\sigma, \lambda)=(-0.5,20)$.

$\theta=0.1<\theta_{3}=0.2004$; then, the NPRNN (47) can achieve exponential stability and the stable trajectories are shown in Figure 3. (3) Figure 1 is an stable enclosed curve about $\sigma$ and $\lambda$ depicted by (24) (more details for Figure 1 is shown in Remark 4). (4) If connection weights $|\sigma|=0.001<|\sigma|_{\text {sup }}=0.0071809$ and $|\lambda|=0.01<|\lambda|_{\text {sup }}=$ 0.0797873 , the parameter-uncertainty NPRNN (48) will be stable again, and the stable trajectories are shown in
Figure 4. (5) However, as long as either $|\sigma|>|\sigma|_{\text {sup }}$ or $|\lambda|>|\lambda|_{\text {sup }}$ is true, system (48) will be unstable, that is, the final-time state value does not converge to the same equilibrium point, which can be seen in Figures 5-8. More importantly, Figures 5-8 are essential and favourable reference frames for demonstrating the robustness of the parameter-uncertainty NPRNN. Furthermore, a brief calculation flowchart of Figures 1-8 is provided in Figure 9. 


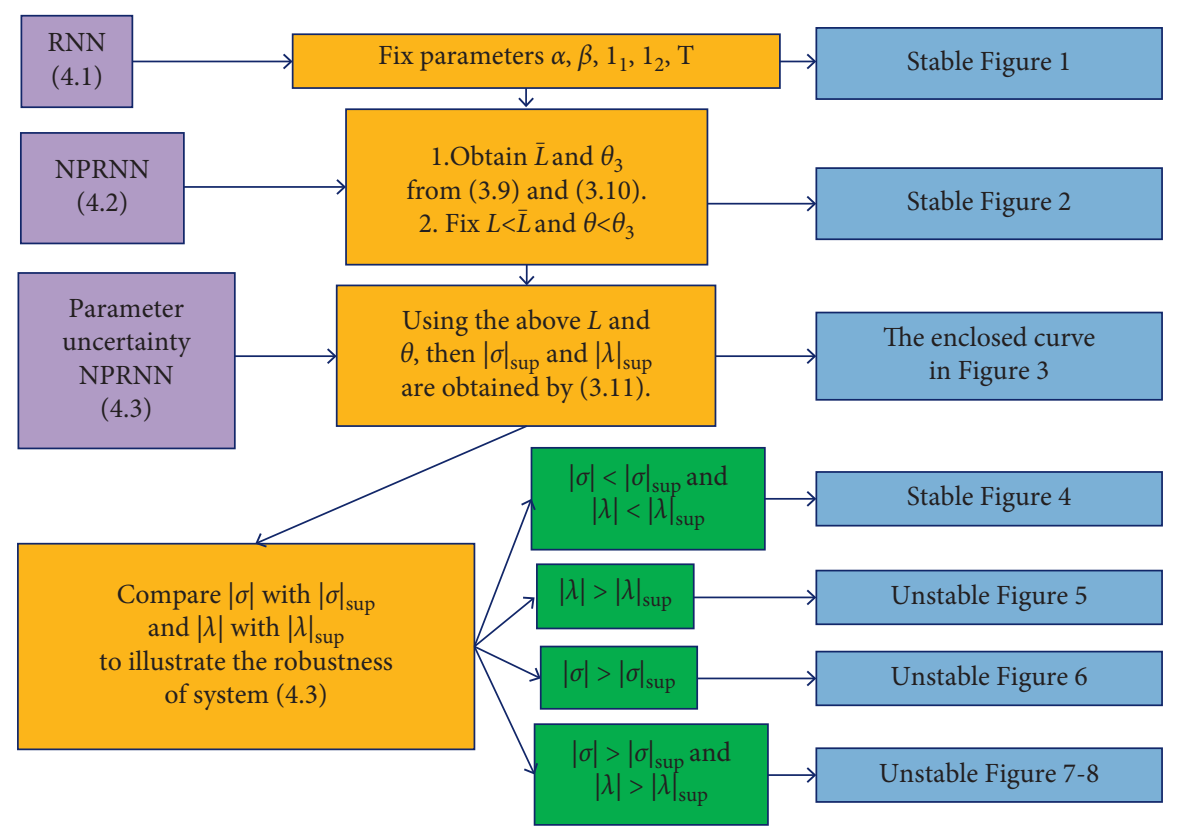

Figure 9: The calculation process of Figures 1-8 in Example 1.

\section{Conclusion}

This paper further explored the robustness of global exponential stability of NPRNN with uncertain dual connection weight matrices. Firstly, a foundational lemma is given. The relationship between the piecewise argument state and the current state is obtained by virtue of Gronwall inequality and the Lipchitz conditions. In addition, a main theorem is given. The upper bounds of the two interference factors and the coupling restrictions of uncertain dual connection weights are deduced by solving a four-element transcendental equation based on stable NPRNN. Finally, the stable and unstable cases of parameter-uncertainty NPRNN are analyzed by a systematic comparison of several numerical simulations, and it is also visually explained that if the two categories of interferences $(L$ and $\theta)$ and dual uncertain connection weights $(\sigma$ and $\lambda$ ) are lower than the deduced values $\left(\bar{L}, \theta_{3},|\sigma|_{\text {sup }}\right.$, and $\left.|\lambda|_{\text {sup }}\right)$ in Theorem 1 , respectively, the disturbed parameter-uncertainty NPRNN based on stable NPRNN can be stabilized again.

Additionally, there is a prospect for future work. On the one hand, future work may focus on the other diverse dynamical stability analysis of NPRNN with connection weight matrices, such as passivity and dissipativity $[3,8]$, multistability [5], and asynchronous analysis [6, 23]. On the other hand, we may also pay attention to attaching more exogenous interferences on the system established in this paper, such as mixed time-varying delays [3], stochastic disturbances [8], and Markov switching [23]. Furthermore, the uncertain-parameter NPRNN can be extended to more multidimensional spaces, such as fractional-order systems [1] and high-order systems [4].

\section{Data Availability}

No data were used to support this study.

\section{Conflicts of Interest}

The authors declare that they have no conflicts of interest.

\section{Acknowledgments}

The authors thank everyone who helped for this paper. Additionally, the authors express their thanks to their school and academy for the fund support. This work was supported by the Natural Science Foundation of China, under Grants 62072164 and 12074111, the Directive Project of Science and Technology Research Plan of Hubei Education Department in 2020, under Grant B2020127, and the Project of Hubei Provincial Education Department in 2021, under Grant BXLBX0536.

\section{References}

[1] Y. Xu, J. Yu, W. Li, and J. Feng, "Global asymptotic stability of fractional-order competitive neural networks with multiple time-varying-delay links," Applied Mathematics and Computation, vol. 389, Article ID 125498, 2021.

[2] Z. Zhang, X. Yang, X. Deng, and L. Li, "A varying-gain recurrent neural-network with super exponential convergence rate for solving nonlinear time-varying systems," Neurocomputing, vol. 351, pp. 10-18, 2019.

[3] R. Saravanakumar, G. Rajchakit, C. K. Ahn, and H. R. Karimi, "Exponential stability, passivity, and dissipativity analysis of generalized neural networks with mixed time-varying delays," IEEE Transactions on Systems, Man, and Cybernetics: Systems, vol. 49, no. 2, pp. 395-405, 2019.

[4] Z. Dong, X. Zhang, and X. Wang, "Global exponential stability of discrete-time higher-order Cohen-Grossberg neural networks with time-varying delays, connection weights and impulses," Journal of the Franklin Institute, vol. 358, no. 11, pp. 5931-5950, 2021.

[5] P. Liu, Z. Zeng, and J. Wang, "Multistability of recurrent neural networks with nonmonotonic activation functions and unbounded time-varying delays," IEEE Transactions on 
Neural Networks and Learning Systems, vol. 29, no. 7, pp. 3000-3010, 2018.

[6] J.-J. Xiong, G.-B. Zhang, J.-X. Wang, and T.-H. Yan, "Improved sliding mode control for finite-time synchronization of nonidentical delayed recurrent neural networks," IEEE Transactions on Neural Networks and Learning Systems, vol. 31, no. 6, pp. 2209-2216, 2020.

[7] Y. Zhou, J. Xia, H. Shen, J. Zhou, and Z. Wang, "Extended dissipative learning of time-delay recurrent neural networks," Journal of the Franklin Institute, vol. 356, no. 15, pp. 8745-8769, 2019.

[8] R. Saravanakumar, H. Mukaidani, and P. Muthukumar, "Extended dissipative state estimation of delayed stochastic neural networks," Neurocomputing, vol. 406, pp. 244-252, 2020.

[9] G. Bao, Y. Peng, X. Zhou, and S. Gong, "Region stability and stabilization of recurrent neural network with parameter disturbances," Neural Processing Letters, vol. 52, no. 3, pp. 2175-2188, 2020.

[10] J. Li, M. Hu, and L. Guo, "Exponential stability of stochastic memristor-based recurrent neural networks with time-varying delays," Neurocomputing, vol. 138, pp. 92-98, 2014.

[11] W. Jiang, L. Li, Z. Tu, and Y. Feng, "Exponential Lagrange stability for impulses in discrete-time delayed recurrent neural networks," International Journal of Systems Science, vol. 50, no. 1, pp. 50-59, 2019.

[12] Q. Chen, L. Liu, and A. Wu, "Mean-square global exponential stability in Lagrange sense for delayed recurrent neural networks with Markovian switching," Neurocomputing, vol. 226, pp. 58-65, 2017.

[13] Y. Jun Wang and J. Wang, "Robustness analysis of global exponential stability of recurrent neural networks in the presence of time delays and random disturbances," IEEE Transactions on Neural Networks and Learning Systems, vol. 23, no. 1, pp. 87-96, 2012.

[14] M. U. Akhmet, D. Aruğaslan, and E. Yılmaz, "Stability analysis of recurrent neural networks with piecewise constant argument of generalized type," Neural Networks, vol. 23, no. 7, pp. 805-811, 2010.

[15] J.-E. Zhang, "Robustness analysis of global exponential stability of nonlinear systems with deviating argument and stochastic disturbance," IEEE Access, vol. 5, pp. 13446-13454, 2017.

[16] Y. He, Q.-G. Wang, and W.-X. Zheng, "Global robust stability for delayed neural networks with polytopic type uncertainties," Chaos, Solitons \& Fractals, vol. 26, no. 5, pp. 1349-1354, 2005.

[17] V. Singh, "Global robust stability of delayed neural networks: estimating upper limit of norm of delayed connection weight matrix," Chaos, Solitons \& Fractals, vol. 32, no. 1, pp. 259-263, 2007.

[18] S. Zhu and Y. Shen, "Robustness analysis for connection weight matrix of global exponential stability recurrent neural networks," Neurocomputing, vol. 101, pp. 370-374, 2013.

[19] S. Zhu and Y. Shen, "Robustness analysis for connection weight matrices of global exponential stability of stochastic recurrent neural networks," Neural Networks, vol. 38, pp. 17-22, 2013.

[20] S. Zhu and Y. Shen, "Robustness analysis for connection weight matrices of global exponential stable time varying delayed recurrent neural networks," Neurocomputing, vol. 113, pp. 220-226, 2013.

[21] S. Zhu, K. Zhong, and Y. Zhang, "Robustness analysis for parameter matrices of global exponential stable stochastic time varying delay systems," Communications in Nonlinear Science and Numerical Simulation, vol. 19, no. 1, pp. 128-138, 2014.
[22] W. X. Si, T. Xie, and B. W. Li, "Exploration on robustness of exponentially global stability of recurrent neural networks with neutral terms and generalized piecewise constant arguments," Discrete Dynamics in Nature and Society, vol. 2021, Article ID 9941881, 13 pages, 2021.

[23] J. Cheng, Y. Wu, L. Xiong, J. Cao, and J. H. Park, "Resilient asynchronous state estimation of Markov switching neural networks: a hierarchical structure approach," Neural Networks, vol. 135, pp. 29-37, 2021.

[24] A. Bellen, N. Guglielmi, and M. Zennaro, "On the contractivity and asymptotic stability of systems of delay differential equations of neutral type," Bit Numerical Mathematics, vol. 39, no. 1, pp. 1-24, 1999.

[25] M. Hui, N. Luo, Q. Wu, R. Yao, and L. Bai, "New results of finite-time synchronization via piecewise control for memristive Cohen-Grossberg neural networks with time-varying delays,” IEEE Access, vol. 7, pp. 79173-79185, 2019.

[26] M. Song, Y. Geng, and M. Liu, "Stability equivalence among stochastic differential equations and stochastic differential equations with piecewise continuous arguments and corresponding Euler-Maruyama methods," Applied Mathematics and Computation, vol. 400, Article ID 125813, 2021.

[27] D. Peaucelle, D. Arzelier, O. Bachelier, and J. Bernussou, "A new robust D-stability condition for real convex polytopic uncertainty," Systems \& Control Letters, vol. 40, no. 1, pp. 21-30, 2000.

[28] Y. Q. Xia and Y. M. Jia, "Robust control of state delayed systems with polytopic type uncertainties via parameter-dependent Lyapunov functionals," Systems \& Control Letters, vol. 50, no. 3, pp. 183-193, 2003.

[29] J. Olejnik and A. Olejnik, "QML estimation with non-summable weight matrices," Journal of Geographical Systems, vol. 22, pp. 469-495, 2020.

[30] F. Corpas-Burgos and M. A. Martinez-Beneito, "On the use of adaptive spatial weight matrices from disease mapping multivariate analyses," Stochastic Environmental Research and Risk Assessment, vol. 34, pp. 531-544, 2020.

[31] J. S. Zhang, J. Y. Hu, and J. M. Liu, "Neural network with multiple connection weights," Pattern Recognition, vol. 107, Article ID 107481, 2020.

[32] N. Rathi, P. Panda, and K. Roy, "STDP-based pruning of connections and weight quantization in spiking neural networks for energy-efficient recognition," IEEE Transactions on Computer-Aided Design of Integrated Circuits and Systems, vol. 38, no. 4, pp. 668-677, 2019.

[33] W. J. Lv, Z. W. Yang, and M. Z. Liu, "Stability of the EulerMaclaurin methods for neutral differential equations with piecewise continuous arguments," Applied Mathematics and Computation, vol. 186, no. 2, pp. 1480-1487, 2007.

[34] C. J. Zhang and Y. Y. He, "The extended one-leg methods for nonlinear neutral delay-integrodifferential equations," Applied Numerical Mathematics, vol. 59, no. 6, pp. 1409-1418, 2009.

[35] C. Li and C. G. Zhang, "Block boundary value methods applied to functional differential equations with piecewise continuous arguments," Applied Numerical Mathematics, vol. 115, pp. 214-224, 2017.

[36] C. J. Zhang, W. S. Wang, B. C. Liu, and T. T. Qin, "A multidomain Legendre spectral collocation method for nonlinear neutral equations with piecewise continuous argument," International Journal of Computer Mathematics, vol. 95, no. 12, pp. 2419-2432, 2018.

[37] C. J. Zhang, C. Li, and J. Y. Jiang, "Extended block boundary value methods for neutral equations with piecewise constant argument," Applied Numerical Mathematics, vol. 150, pp. 182-193, 2020. 